\title{
Luxação lateral de patela em um Poodle toy
}

\author{
Lateral patellar luxation in Poodle toy \\ Ísis dos Santos Dal-Bó, Márcio Poletto Ferreira, Marcelo Meller Alievi, Luciana Machado \\ da Silva, Fernanda Silveira Nóbrega, Paula Cristina Sieczkowski Gonzalez \\ \& Carlos Afonso de Castro Beck
}

\begin{abstract}
Background: Luxating patella is one of the most frequent conditions of the stifle joint in dogs and it can evolve into a degenerative joint disease. The occurrence of the condition can be medial or lateral, unilateral or bilateral. Its origin can be traumatic or congenital and, additionally, it may undergo genetic and environmental influence. Lateral luxation is more common in large breed dogs, and it can rarely affect medium and small breeds, whilst medial luxation is more common in small breed dogs. The classification of patellar luxation comprises four grades, according to the patellar position, clinical signs and seriousness of the bone deformities found. The diagnosis is made based on the clinical evaluation, associated with a radiographic evaluation. The present study aims at describing a case of bilateral grade 3 lateral luxating patella in a Toy Poodle dog. Case: A five-month-old female Toy Poodle dog was presented for difficulty standing and claudication of hind limbs. Orthopedic evaluation revealed internal rotation of the stifle, claudication of hind limbs during walking and "jumps" during trotting, in addition to a lateral grade 3 luxating patella and slight restriction of the joint motion upon extension of the right and left hind limbs. Radiographic evaluation of both stifles evidenced an ectopic (lateral) position of the right and left patellas. The animal was referred to the surgery department, where it was submitted to surgical intervention of both stifles. A reduction of luxation in both stifles was achieved through medial arthrotomy, wedge recession trochleoplasty, lateral desmotomy and medial imbrication of the articular capsule techniques. Tramadol hydrochloride, dipyrone and meloxicam were prescribed as postoperative medication. Daily wound care, resting and mild walks using a lead were recommended for the animal. On the tenth postoperative day, the stitches were removed from the skin and the animal seemed to be in a good general state, using adequately its hind limbs when walking and trotting.

Discussion: The medial form of the congenital patellar luxation is common both in large and small breeds. However, the lateral form is more common in large or giant breeds, differently to the findings of the present case, in which luxation occurred in a Toy Poodle. Surgery is recommended in animals with a luxating patella grade starting from 2, as even the smallest luxation grades may evolve into higher grades, causing functional inability of the limb and degenerative joint disease. Surgery was justified in this case, because the animal presented a grade 3 luxating patella. The decision on which technique should be used will depend on the luxating patella grade and on soft tissue compromise. The most recommended procedure is a combination of various techniques in the same articulation. In this case, the use of wedge recession trochleoplasty and medial imbrication of the articular capsule techniques was successful, as a few days after surgery the animal was able to walk adequately without presenting any instability of the patellas, thus improving its quality of life. Moreover, upon reaching adulthood, the animal maintained an adequate gait, without excessive patellar motion.
\end{abstract}

Keywords: stifle, dog, trochleoplasty, orthopedics.

Descritores: joelho, cão, trocleoplastia, ortopedia. 


\section{INTRODUÇÃO}

A luxação de patela é uma das doenças da articulação fêmoro-tíbio-patelar mais frequentes em cães e pode resultar no desenvolvimento de doença articular degenerativa $[1,3]$. A etiologia da luxação de patela pode ser congênita ou traumática e a apresentação pode ser uni ou bilateral [2]. A luxação patelar congênita (medial ou lateral) sofre influências genéticas e ambientais [1,4,7]. A luxação lateral é mais comum em cães de raças grandes, podendo acometer, raramente, raças médias e pequenas [4], enquanto a luxação medial é mais comum em raças pequenas [2].

A fisiopatologia do deslocamento da patela ainda não está esclarecida [7], no entanto, a luxação lateral está associada a anormalidades anatômicas como aumento do ângulo de anteversão do colo femoral, articulação fêmoro-tíbio-patelar valga, torção medial distal do fêmur, hipoplasia do músculo vasto medial, torção lateral da tíbia e displasia coxofemoral [4,6].

A luxação patelar pode ser classificada em quatro graus, sendo que no grau um a luxação é apenas um achado durante o exame ortopédico. No grau dois, pode-se realizar luxação manual, mas a mesma é reduzida durante a flexão e extensão do joelho. No grau três, a patela permanece luxada a maior parte do tempo, podendo ser reduzida manualmente, entretanto, retorna à posição luxada e, no grau quatro, a patela se encontra luxada permanentemente, não podendo ser reposicionada. As alterações na marcha, as anormalidades musculares e deformidades angulares e rotacionais em fêmur e tíbia são proporcionais ao grau de luxação [2].

Os sinais clínicos variam de acordo com o grau de luxação e incluem claudicação, defeitos conformacionais, dor e relutância em se mover. O diagnóstico é realizado através do exame ortopédico, contudo, a avaliação radiográfica é imprescindível para determinar o grau de deformidade do membro e o grau de doença articular degenerativa [7].

O presente relato objetiva descrever um caso de luxação lateral de patela grau três bilateral em um cão da raça Poodle toy.

\section{RELATO DE CASO}

Um canino, fêmea, da raça Poodle toy, com cinco meses de idade foi atendido no Hospital de
Clínicas Veterinárias da Universidade Federal do Rio Grande do Sul (HCV-UFRGS), com histórico de dificuldade para se manter em estação e claudicação com apoio dos membros pélvicos. Ao exame ortopédico, observou-se rotação interna dos joelhos ( $g e$ nu valgum), claudicação dos membros pélvicos durante a marcha e "saltos" durante o trote. À palpação de ambas as articulações fêmoro-tíbio-patelares foi observada luxação lateral de patela grau três e leve restrição da amplitude de movimento articular à extensão.

O paciente foi submetido à avaliação radiográfica de ambos os joelhos, sendo verificada a posição ectópica (lateral) das patelas direita e esquerda (Figura 1-A) e a ausência de outras alterações articulares ou ósseas. O cão foi encaminhado para o setor de cirurgia, onde foi pré-medicado com maleato de acepromazina $^{1}\left(0,05 \mathrm{mg} \cdot \mathrm{kg}^{-1}\right)$ e cloridrato de meperidina $^{2}\left(3 \mathrm{mg} \cdot \mathrm{kg}^{-1}\right)$, após 15 minutos, a indução anestésica foi realizada com propofol ${ }^{3}\left(4 \mathrm{mg} \cdot \mathrm{kg}^{-1}\right)$ e a manutenção anestésica foi feita com isofluorano $\mathrm{em}^{4}$ oxigênio a $100 \%$ em sistema aberto.

O paciente foi submetido à intervenção cirúrgica em ambos os joelhos, onde foram realizadas após artrotomia medial, trocleoplastia em cunha (Figura 1-B), desmotomia lateral e imbricação da cápsula articular medial com padrão de sutura Sultan e fio de mononáilon 2-0 (Figura 1-C), reduzindo as luxações. A musculatura e o tecido subcutâneo foram aproximados com fio de poliglactina 910 3-0 e padrão simples contínuo e a dermorrafia foi realizada com padrão isolado simples e fio de mononáilon 3-0. Como medicações pós-operatórias, foram prescritos cloridrato de tramadol ${ }^{6}\left(3 \mathrm{mg} \cdot \mathrm{kg}^{-1}\right)$, dipirona sódica $^{7}\left(25 \mathrm{mg} \cdot \mathrm{kg}^{-1}\right)$ e meloxicam ${ }^{8}\left(0,1 \mathrm{mg} \cdot \mathrm{kg}^{-1}\right)$. O proprietário foi orientado a manter o animal em espaço restrito, com repouso e caminhadas leves com guia.

Após 10 dias da cirurgia, o animal retornou ao hospital para retirada dos pontos de pele e reavaliação clínica, apresentando-se em bom estado geral e utilizando adequadamente os membros pélvicos durante a marcha e o trote. Durante a palpação dos joelhos, pôde-se constatar que não mais havia mobilidade lateral das patelas e o animal recebeu alta clínica.

\section{RESULTADOS E DISCUSSÃO}

A luxação congênita de patela é comum na forma medial tanto em raças grandes quanto em pequenas, entretanto, na forma lateral é mais comum 
nas raças grandes ou gigantes [2], diferente do encontrado neste caso, onde a luxação lateral de patela ocorreu em um Poodle Toy. Em um estudo retrospectivo que avaliou luxações de patela em cães [7], foi observado que dos animais portadores de luxação de patela com raça definida, houve prevalência de animais da raça Poodle, porém a maioria com luxação medial.

A cirurgia é aconselhável em animais que apresentem luxação patelar a partir do grau dois, pois mesmo os graus mais baixos de luxação podem evoluir para graus mais elevados e para doença articular degenerativa e impotência funcional do membro [7]. Nesse caso, havia indicação cirúrgica pelo grau três de luxação e pelos sinais clínicos apresentados pelo animal.

Existem numerosas técnicas para restringir a patela no sulco troclear como trocleoplastia [3], desmotomia, imbricação da cápsula articular, sutura antirrotacional e as osteotomias tibiais e femorais [2]. A decisão sobre qual das técnicas deve ser utilizada depende do grau de luxação patelar e do comprometimento dos tecidos moles [5]. O mais recomendado é a combinação de várias técnicas em uma mesma articulação e a escolha pelas osteotomias a partir do grau três [7]. Embora possam ser realizado, além da trocleoplastia, osteotomia corretiva da crista da tíbia [6], no presente caso não foram realizadas osteoto- mias em virtude do pequeno grau de deformidade angular e rotacional encontrado nas radiografias. Em animais com mais de $20 \mathrm{~kg}$ e que não são submetidos a osteotomias corretivas, há maior probabilidade de complicações cirúrgicas (reluxação patelar) [5].

\section{CONCLUSÃO}

Neste relato, as técnicas de trocleoplastia em cunha e de imbricação da cápsula articular medial apresentaram sucesso, pois poucos dias após a cirurgia, o animal conseguia deambular adequadamente sem qualquer instabilidade nas patelas, melhorando assim a sua qualidade de vida e ao atingir a idade adulta permanecia com deambulação adequada e sem mobilidade excessiva das patelas.

\section{NOTAS INFORMATIVAS}

${ }^{1}$ Acepran ${ }^{\circledast}$ - Univet S/A - Indústria Veterinária. São Paulo, SP.

${ }^{2}$ Dolosal $^{\circledR}$ - Cristália, rod. Itapira, km 14, Itapira, SP, Brasil. ${ }^{3}$ Diprivan $^{\circledR}$ - Laboratórios Wellcome - ICI Ltda. Cotia, SP. ${ }^{4}$ Forane $^{\circledR}$ - Abbott, Rua Nova York, 245, São Paulo, SP, Brasil.

${ }^{5}$ Vicryl $^{\circledR}$, Ethicon, São José dos Campos, São Paulo, SP

${ }^{6}$ Tramal $^{\circledR}$ - Cristália, rod. Itapira, km 14, Itapira, SP, Brasil. ${ }^{7}$ Dipirona sódica ${ }^{\circledR}$ - Medley, Rua Macedo Costa, 55, Campinas, SP, Brasil.

${ }^{8}$ Meloxivet $^{\circledR}$ - Duprat, Rua Almirante Oliveira Pinto, 267, Rio de Janeiro, RJ, Brasil.

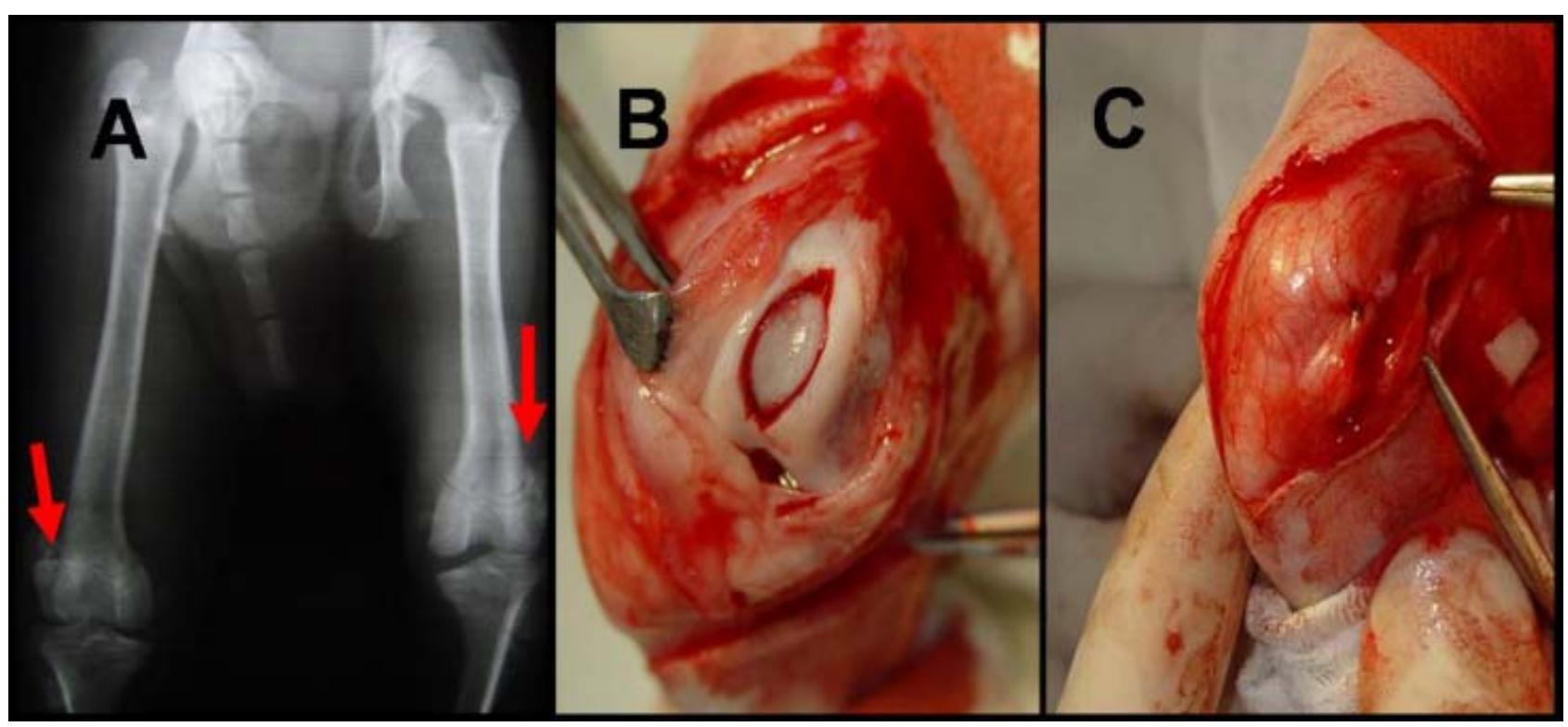

Figura 1. A - Exame radiográfico das articulações fêmoro-tíbio-patelares (FTP) na projeção crânio-caudal evidenciando deslocamento patelar lateral em ambos os joelhos (setas vermelhas). B - Artrotomia medial e trocleoplastia em cunha na articulação FTP direita. C - Imbricação do retináculo na articulação FTP direita. 


\section{REFERÊNCIAS}

1 Bound N., Zadai D., Butterworth S.J. \& Pead M. 2009. The prevalence of canine patellar luxation in three centers - clinical features and radiographic evidence of limb deviation. Veterinary Comparative Orthopaedics and Traumatology. 22(1): 32-37.

2 Hulse D.A. \& Johnson A.L. 2005. Artropatias. In: Fossum, T.W. (ed) Cirurgia de pequenos animais. 2.ed. São Paulo: Roca, pp. 1017-1048.

3 Johnson A.L., Probst C.W., Decamp C.E., Rosenstein D.S., Hauptman J.G., Weaver B.T. \& Kern T.L. 2001. Comparison of trochlear block recession and trochlear wedge recession for canine patellar luxation using a cadaver model. Veterinary Surgery. 30(2): 140-150.

4 L'eplattenier H. \& Montavon P. 2002. Patellar luxation in dog and cats: patogenesis and diagnosis. Compendium on Continuing Education for the Practicing Veterinarian. 24 (Suppl 3): 234-239.

5 MostafaA.A., Griffon D.J., Thomas M.W. \& Constable P.D. 2008. Proximodistal alignment of the canine patella: radiographic evaluation and association with medial and lateral patelar luxation. Veterinary Surgery. 31(3): 201-211.

6 Padilha Filho J.G., Dórea Neto F.A., Dórea H.C., Sanches R.C. \& Canola J.C. 2005. Treatment of the lateral patellar luxation in toy poodles. Ciência Rural. 25 (Supl 4): 843-847.

7 Souza M.M.D., Rahal S.C., Otoni C.C., Mortari A.C. \& Lorena S.E.R.S. 2009. Luxação de patela em cães: estudo retrospectivo. Arquivo Brasileiro de Medicina Veterinária e Zootecnia. 61 (Supl 2): 523-526. 